\title{
Baryonic Dark Matter in the Milky Way
}

\author{
Peter M.W. Kalberla* \\ RAIUB, Germany \\ E-mail: pkalberla@astro.uni-bonn.de
}

We study the question whether some of the dark matter in the Milky Way may be baryonic. Considering a proposal by Pfenniger \& Combes that dark matter might be associated with the Galactic disk, we study first the case whether such a model may be consistent at all.

To model the mass distribution in the Milky Way we assume that dark matter may be contained in an isothermal sphere but also within the disk. We add bulge, bar, and two stellar disks, thick and thin. The gas is characterized by a molecular component, HI gas (cold and warm neutral medium), a diffuse ionized gas component, and a more extended halo component giving rise to the soft X-ray background, but also observable as faint HI lines. All disk components are assumed to be isothermal.

A self-consistent solution of the Poisson-Boltzmann equation for mass distribution and gravitational potential in 3-D is discussed. We find a pronounced flaring for all of the isothermal disk components. The flaring derived from the model is in excellent agreement with observations. Oort limit and the local gravitational potential perpendicular to the plane agree with observations. In general, such a solution is consistent with all known observational constraints.

The proposal that a disk-like dark matter component in the Milky Way exists in form of $\mathrm{H}_{2}$ clumpuscules is found to be very appealing. A "conspiracy" between gas and dark matter would be a natural consequence. Cloud-cloud collisions and evaporation of $\mathrm{H}_{2}$ clumpuscules would explain that we can observe an $\mathrm{X}$-ray background and faint $\mathrm{HI}$ emission lines despite the fact that the cooling timescale is similar to the free fall timescale. The turbulent motion of the clumpuscules would be important for the stability of the Milky Way. At the same time turbulence would explain the scale height of the gaseous halo.

Such a baryonic dark matter disk would be consistent with the expected baryon fraction. Similar, the angular momentum of the Milky Way disk would get easily explainable.

Baryons in Dark Matter Halos

5-9 October 2004

Novigrad, Croatia

${ }^{*}$ Speaker. 


\section{Introduction}

There is no doubt that dark matter (DM) dominates the mass distribution within the local group but only little is known about the details of its distribution closer to the Galactic disk. After some 40 years of DM research in the Milky Way, based on stellar dynamics, a general agreement exists on the boundary conditions of the DM distribution: 1) there is little dark matter associated with the stellar component of the Galactic disk and 2) the total mass within $350 \mathrm{kpc}$, half-ways to M31, is $M \sim 2 \cdot 10^{12} \mathrm{M}_{\odot}$, several times the mass of the stellar component. The standard assumption is that the DM distribution may be modeled as an isothermal sphere with a core radius of a few kpc (e.g. [4]).

It was noted, however, more than 20 years ago that for many galaxies the DM distribution appears to be linked to the large scale distribution of gas, there seems to be a "conspiracy" between gas and mass. Since the observed gas on its own can hardly provide signifi cant amounts of mass, it is appealing to speculate whether dark matter can be associated with the gaseous disk. Pfenniger \& Combes [2] proposed that this mass may be in the form of cold molecular gas. Such a phase would condense to clumps with diameters of a few AU and would be hardly observable (see Pfenniger, these proceedings [5]).

In our contribution we study the question whether a disk-like DM distribution may exist and how far this distribution can be constraint from observations. These considerations are completely independent of the nature of the DM distribution but, as we shall see later, hint to $\mathrm{H}_{2}$ clumpuscules as proposed by Pfenniger \& Combes.

\section{Dark matter in the Milky Way disk - can it be?}

To test the hypothesis of a conspiracy between dark matter and gas we assume that there might be a DM disk but we do not question that on large scales there might be in addition a DM distribution that can be described best by a sphere. For simplicity we assume both distributions to be isothermal.

We characterize the observable mass distribution by a central bulge, a bar, and two stellar disks, thick and thin. The gas, most important as a tracer of the potential, is characterized by a molecular component, HI gas (cold and warm neutral medium), a diffuse ionized gas component, and a more extended halo component giving rise to the soft X-ray background, but also observable as a faint HI distribution (e.g. Dedes, these proceedings [1]).

We assume the disk components to be isothermal and characterize the distribution by the radial scale length, the local scale height and density. Parameters are readily available in the literature.

To build up a mass model we solve the combined Poisson-Boltzmann Equation in a selfconsistent way [3]. For the mass distribution, assumed initially to be plane-parallel, we calculate the corresponding gravitational potential. In the next step the potential is inserted into the Boltzmann equation, refi ning this way the distribution perpendicular to the Galactic plane. We notice that the resulting mass distribution differs from the initial estimate. In the inner Galaxy the scale heights of our disk components decrease, the opposite happens at large galactocentric radii. We use these results to calculate a refi ned potential which is inserted again into the Boltzmann equation. This 
process is repeated until convergence. About 8 cycles are needed. For all of the iterations we tune the model parameters such that the observational constraint are satisfi ed.

The pronounced fhring of individual isothermal disk components is one of the most striking results from our calculation. Scale heights of the inner and outer parts of the disks differ by typically a factor of ten. A plane-parallel layer, assumed in most mass models, is found to be inconsistent with a self-consistent solution of the Poisson-Boltzmann Equation and is possible only in case of strongly non-isothermal disks. In this case the temperature needs to drop an order of magnitude from the inner to the outer parts of the disk. This appears inconsistent with observations.

Fig. 1 shows to the left a comparison between model and observed faring of the HI layer. Please note that the scale height of the model is constraint only at a single point, at $\mathrm{R}_{\odot}=8.5 \mathrm{kpc}$. On the right we plot the scale height of the corresponding DM disk (top) and show how flaring of the disk builds up during iterations 1 to 4 (from bottom up).
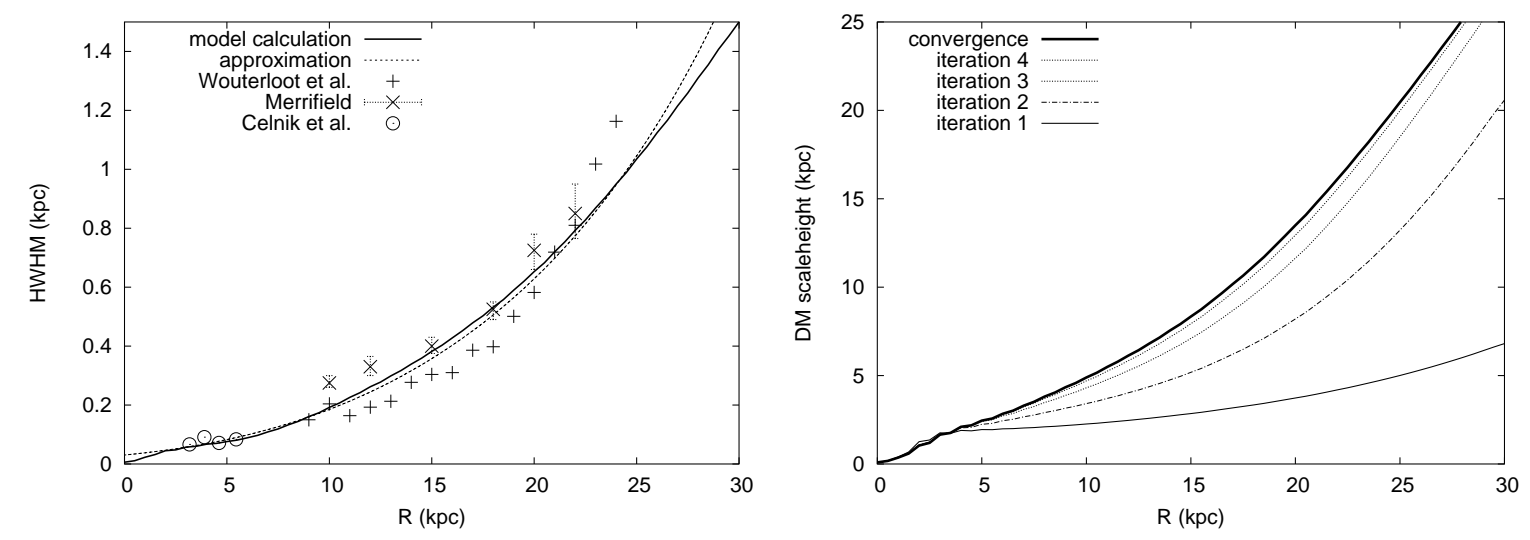

Figure 1: Left: flaring of the observed HI gas layer in comparison with the model. Right: flaring of the DM disk (top curve). The lower curves show how during the iterations 1 to 4 the self-consistent model builds the flaring up (from bottom to top).

Some more details of the mass model appear worth to be mentioned: Stars as well as gas components organize themselves independently as exponential disks. At the same time hollow cores and nasty imaginary rotational velocities, as found frequently for the gaseous disks in mass models, disappear after a few iterations. The interplay between gas and DM disk is such that they couple in the outer part of the disk, explaining "conspiracy" between gas and dark matter. The dark matter disk is stable. Both, the Toomre and the Ostriker \& Peebles criteria are satisfi ed. Last, and not least, the observed scale heights of the gaseous halo match the model closely. Note a signifi cant difference to most of the other mass models: the gas in our model is not self-gravitating. In particular, the halo gas is a tracer of the potential only, its mass is negligible.

Temporary astronomy textbooks teach students that there is no evidence for dark matter associated with the Galactic disk. Two arguments are brought as a proof: 1) The local dynamical mass volume density, the Oort limit, agrees with the observed density. No additional mass is needed locally. 2) Gravitational potential and stellar density distribution perpendicular to the plane doesn't need to be explained by an additional mass hidden in the disk.

These arguments are correct, but incomplete. Observations tell us only that there must be a lower limit to the scale height of any dark matter component within the disk. We derive a local 
scale height of $4 \mathrm{kpc}$ for a DM disk component. Such a mass model is in full agreement with any observational constraint, in particular with the Oort limit and the gravitational potential derived by Kuijken \& Gilmore [4].

\section{A Milky Way dark matter disk - can it be $\mathrm{H}_{2}$ ?}

We have argued in the previous section that a fhring dark matter disk is consistent with all known observational constraints. Does such a model provide further advantages?

The mass model, considered so far, doesn't tell us anything about the nature of the DM disk. It is, however, appealing to adopt the hypothesis that dark matter in the Milky Way may be in the form of $\mathrm{H}_{2}$ clumpuscules. The only modifi cation to the proposal by Pfenniger \& Combes would be the necessity to have a local scale height of $4 \mathrm{kpc}$. Such a scale height explains the observed fhring best (see Fig. 1). At the same time it provides just the right ratio between kinetic and rotational energy to stabilize a disk. The conspiracy between derived dark matter and observed HI gas contribution would get a natural explanation. EGRET $\gamma$-ray observations constrain the diameter of the $\mathrm{H}_{2}$ clumpuscules to a few $\mathrm{AU}$ [2]. Cloud-cloud collisions and evaporation of $\mathrm{H}_{2}$ clumps can provide the necessary continuous support for a gaseous halo, needed to explain both, the soft Xray background at a temperature of $10^{6.15} \mathrm{~K}$ and the cold halo $\mathrm{HI}$ gas. In addition, we get an explanation for the observed scale height of the gaseous halo and the velocity dispersion of the HI component.

A baryonic dark matter disk is also appealing for other reasons: Such a mass model implies that $13 \%$ of the mass within $350 \mathrm{kpc}$ is in baryonic. This is about the number we get from nucleosynthesis. For the angular momentum we obtain roughly the same fraction. $15 \%$ of the angular momentum in the Milky Way would be associated with baryons, solving the angular momentum problem for the Milky Way. Predictions from $\lambda$ CDM models deviate by more than a factor 10 from observations.

\section{References}

[1] Dedes, L. Spiral structure and the clumpy HI Sub-Structure of the Halo of the Milky Way in proceedings of Baryons in Dark Matter Halos, Eds R-J., Dettmar, U. Klein, P. Salucci, PoS, SISSA, PoS(BDMH2004)019

[2] Kalberla, P. M. W., Shchekinov, Y. A., \& Dettmar, R.-J. $H_{2}$ dark matter in the galactic halo from EGRET 1999, A\&A, 350, L9

[3] Kalberla, P. M. W. 2003, Dark Matter in the Milky Way. I. The Isothermal Disk Approximation ApJ, 588,805

[4] Kuijken, K., Gilmore, G., The mass distribution in the galactic disc. I - A technique to determine the integral surface mass density of the disc near the sun. II - Determination of the surface mass density of the galactic disc near the sun. III - The local volume mass density 1989, MNRAS 239, 571

[5] Pfenniger, D. \& Combes, F. Is dark matter in spiral galaxies cold gas? II. Fractal models and star non-formation 1994, A\&A, 285, 94

[6] Pfenniger, D., Dark molecular hydrogen in proceedings of Baryons in Dark Matter Halos, Eds R-J., Dettmar, U. Klein, P. Salucci, PoS, SISSA, PoS(BDMH2004)087 